\title{
Professor Dr. sc. med. Walter Schilling (14. Juli 1932-7. April 2014)
}

Walter Schilling, Direktor und Chefarzt der Pneumologischen Klinik des Forschungsinstitutes für Lungenkrankheiten und Tuberkulose (FLT) in Berlin-Buch von 1979 bis 1991 (heute Evangelische Lungenklinik Berlin [ELK]), ist am 7. April 2014 nach langer schwerer Krankheit verstorben. Die Autoren möchten mit diesem Nachruf an seine ethische Einstellung als Arzt und seine Arbeit als Kliniker und Lehrer, aber auch an seine Aufgaben, die ihm mit der Übernahme des Institutes oblagen, erinnern.

Sowohl in der studentischen Ausbildung an der Charité als auch an der Akademie für Ärztliche Fortbildung hat er für die weitere Vertiefung der Kenntnisse auf unserem Fachgebiet Pneumologie Sorge getragen.

Sein besonderes klinisches Interesse galt den Untersuchungen zur pulmonalen Hypertonie, die er im FLT mit dessen kompletter personellen und technischen Ausstattung fortsetzen konnte. Er vertiefte auch die Zusammenarbeit des FLT mit Einrichtungen der biomedizinischen Grundlagenforschung, so zur Rolle von Neuropeptiden (insbesondere Substanz P) in der Pathogenese pulmonaler Erkrankungen im Rahmen einer gemeinsamen Arbeitsgruppe mit dem damaligen Institut für Wirkstoffforschung (IWF) der Akademie der Wissenschaften.

Unter seiner Leitung wurde die von Paul Steinbrück eingeleitete Umwandlung des Fachgebietes mit der Fokussierung auf neue Schwerpunkte innerhalb des Spektrums der Atemwegserkrankungen konsequent weitergeführt. Das bezog auch gesundheitspolitische Fragen ein, deren Lösungen erforderlich waren (z. B. Neuordnung der gesetzlichen Grundlagen für die Volksröntgenreihenuntersuchungen und für die BCG-Impfung). Gemeinsam mit den zuständigen Arbeitsgruppen der damaligen Gesellschaft für Bronchopneumonologie, deren Präsident Walter Schilling von 1985 bis 1988 war, wurden unter Federführung des FLT auch Gesetzesvorlagen erarbeitet und

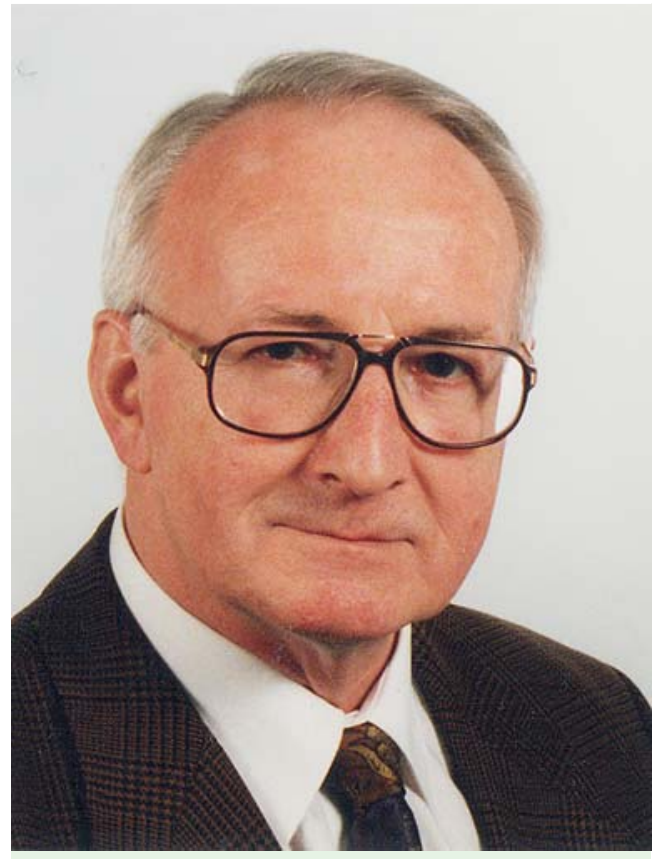

Prof. Dr. sc. med. Walter Schilling

umgesetzt. Als Ausdruck der Wertschätzung wurden ihm Ehrenmitgliedschaften auch internationaler Fachgesellschaften verliehen.

Nach der langjährigen engen Zusammenarbeit mit der IUATLD wurde in Anerkennung der erfolgreichen Tuberkulosebekämpfungsmaßnahmen und der weiteren Entwicklung des Fachgebietes das Generalsekretariat für die europäische Region im FLT Berlin-Buch 1987 etabliert und Walter Schilling zu deren Generalsekretär ernannt.

Mit Walter Schilling verliert die Pneumologie in Deutschland einen Kollegen, der sich über viele Jahre engagiert und erfolgreich für unser Fachgebiet eingesetzt hat.

\section{Grohé, P. Oehme, B. Wiesner}

\section{Bibliografie}

Dol http://dx.doi.org/ 10.1055/s-0034-1365744

Pneumologie 2014; 68: 432 (c) Georg Thieme Verlag KG Stuttgart · New York ISSN 0934-8387

\section{Korrespondenzadresse}

\section{Prof. Dr. Christian Grohé}

Ev. Lungenklinik Berlin Klinik für Pneumologie Lindenberger Weg 27 13125 Berlin

Christian.Grohe@elk-berlin.de 